\title{
Carleson measure and monogenic functions
}

\author{
by \\ S. Bernstein (Freiberg) and P. Cerejeiras (Aveiro)
}

\begin{abstract}
We present necessary and sufficient conditions for a measure to be a $p$ Carleson measure, based on the Poisson and Poisson-Szegô kernels of the $n$-dimensional unit ball.
\end{abstract}

1. Introduction. The study of $H^{p}$-theory started in 1915 with the work of G. Hardy on the mean value of an analytic function on the unit disk. It soon proved to have a deep connection with $L_{p}$-boundary results and it required techniques of both real and complex analysis. Attempts to extend this theory to higher dimensions began in the seventies and coincided with the discovery of the identification of the dual of $H^{1}$ with BMO, the space of functions of bounded mean oscillation. The important result that $\varphi \in$ BMO iff its harmonic extension $u$ to the unit ball has the property that $|\nabla u|^{2}(1-|z|) d x d y$ is a Carleson measure (C. Fefferman) shows a close relation between this space and Carleson measures.

For a higher dimensional counterpart there are several problems arising. In the first place, the group of automorphisms of the unit ball (a Lie group) no longer preserves the Laplace operator, but instead, it preserves the LaplaceBeltrami operator. Thus, the conformally invariant kernel is no longer the Poisson kernel, but the Poisson-Szegó kernel. Second, there exist two possible generalizations to higher dimensions of the classical complex function theory, both dating from the 30's: 1) the several complex variables' approach which has the strong drawback that the Cauchy kernel strongly depends on the shape of the domain under consideration, 2) the Clifford analysis' approach which started with the work of Fueter (1934) on quaternions and later on was developed by the group led by Delanghe to deal with the universal real

2000 Mathematics Subject Classification: Primary 30G35; Secondary 31B10, 30C40.

Key words and phrases: Carleson measure, Poisson kernel, Poisson-Szegó kernel.

Research of P. Cerejeiras partially supported by Unidade de Investigação Matemática e Aplicações of Universidade de Aveiro, through Programa Operacional "Ciência, Tecnologia, Inovação" (POCTI) of the Fundação para a Ciência e a Tecnologia (FCT), cofinanced by the European Community fund FEDER. 
Clifford algebras $\mathcal{C}_{p, q}$. It presents the advantage of providing a Cauchy kernel independent of the domain and a series expansion, the so-called CauchyKovalevskaya extension, which is in general linked to the Euclidean metric. Thus, in order to consider Carleson measures in a higher dimensional setting we are led to an approach via Clifford analysis with an extra consideration of the tensor metric effect on the arising reproducing kernels.

The layout of the paper is as follows: in Section 2 we present some basic definitions and results concerning Clifford analysis. Section 3 is dedicated to a short review of the automorphic group of the $n$-dimensional unit ball, followed by a description of the main properties of the Poisson, PoissonSzegó and Szegó kernels in Section 4. Finally, in Section 5 we shall consider $p$-Carleson measures, together with necessary and sufficient conditions for a measure to be a $p$-Carleson measure.

\section{Clifford analysis}

2.1. Clifford algebras. Let $e_{1}, \ldots, e_{n}$ be an orthonormal basis of the real vector space $\mathbb{R}^{n}$ equipped with the negative definite quadratic form,

$$
Q(x)=-|x|^{2},
$$

where $|\cdot|$ denotes the standard Euclidean norm in $\mathbb{R}^{n}$. We define the universal real-valued Clifford algebra $\mathrm{Cl}_{0, n}$ as the $2^{n}$-dimensional associative algebra generated by $\mathbb{R}^{n}$ modulo the quadratic form $Q(x)$. That is, in the algebra $\mathrm{Cl}_{0, n}$ we have the anticommutation relations

$$
e_{i} e_{j}+e_{j} e_{i}=-2 \delta_{i j}
$$

and a basis for $\mathcal{C}_{0, n}$ consists of the identity $e_{0}=1$, together with the elements of the form $e_{A}=e_{i_{1}} \cdots e_{i_{k}}$, where $A=\left\{\left(i_{1}, \ldots, i_{k}\right): 1 \leq i_{1}<\cdots<i_{k} \leq n\right\}$. Hence, each element $a \in \mathcal{C} \ell_{0, n}$ can be written as a linear combination of the basis elements $a=\sum_{A} a_{A} e_{A}, a_{A} \in \mathbb{R}$. Henceforward, we will identify $\mathbb{R}^{n}$ with the linear subspace $\mathbb{R}^{0, n} \subset \mathcal{C l}_{0, n}$ spanned by $e_{1}, \ldots, e_{n}$.

We define an anti-automorphism called conjugation (or main anti-involution) in $\mathrm{Cl}_{0, n}$ by its action on the basis elements:

$$
\bar{e}_{0}=e_{0}, \quad \bar{e}_{j}=-e_{j}, \quad j=1, \ldots, n, \quad \overline{e_{i_{1}} e_{i_{2}} \ldots e_{i_{l}}}=\bar{e}_{i_{l}} \cdots \bar{e}_{i_{1}} .
$$

In particular, for a vector $x \in \mathbb{R}^{n}$ we have $\bar{x}=x_{1} \bar{e}_{1}+\cdots+x_{n} \bar{e}_{n}=-x$ and $x \bar{x}=-x^{2}=|x|^{2}$, the square of the Euclidean norm of $x$.

2.2. Clifford analysis. Clifford analysis offers a function theory which is a higher dimensional analogue to the theory of holomorphic functions of one complex variable (see e.g. [6]).

Let $\Omega$ denote an open region of $\mathbb{R}^{n}$. Properties such as continuity, differentiability, integrability, and so on, are ascribed to a Clifford-valued function 
$f=\sum_{A} e_{A} f_{A}: \Omega \rightarrow \mathcal{C}_{0, n}$ by requiring that all its real-valued components $f_{A}$ should possess them.

A Clifford-valued function $f: \Omega \rightarrow \mathcal{C}_{0, n}$ is called left monogenic (right monogenic, or two-sided monogenic) in $\Omega$ if $D f=0(f D=0$, or $D f=$ $f D=0$, respectively). Here, $D$ denotes the Dirac operator

$$
D f=\sum_{j=1}^{n} e_{j} \frac{\partial f}{\partial x_{j}}
$$

An important function which is both left and right monogenic is the generalized Cauchy kernel

$$
e(x)=\frac{1}{\omega_{n}} \frac{\bar{x}}{|x|^{n}}
$$

where $\omega_{n}$ denotes the surface area of the unit sphere in $\mathbb{R}^{n}$.

The higher dimensional Cauchy's theorem and Cauchy's integral formula are as follows:

Theorem 2.1 (Cauchy's Theorem). Let $\mathcal{B}^{n}$ denote the open unit ball in $\mathbb{R}^{n}$. If $f$ and $g$ are right and left monogenic in a neighborhood of $\overline{\mathcal{B}^{n}}$, then

$$
\int_{\mathcal{S}^{n-1}} f(y) n(y) g(y) d \mathcal{S}_{y}^{n-1}=\int_{\mathcal{B}^{n}}\left[\left(f D_{y}\right)(y) g(y)+f(y)\left(D_{y} g\right)(y)\right] d y=0
$$

where $d \mathcal{S}_{y}^{n-1}$ is the surface element of the boundary $\mathcal{S}^{n-1}$ of $\mathcal{B}^{n}, n(y)$ is the exterior unit normal to $\mathcal{S}^{n-1}$, defined for almost all $y \in \mathcal{S}^{n-1}$, and $d y$ is the volume element of the unit sphere.

Corollary 2.2 (Cauchy's Integral Formula). Under the previous conditions,

$$
\begin{aligned}
& C_{r} f(x)=\int_{\mathcal{S}^{n-1}} f(y) n(y) e(x-y) d \mathcal{S}_{y}^{n-1}= \begin{cases}f(x), & x \in \mathcal{B}^{n} \\
0, & x \notin \overline{\mathcal{B}^{n}}\end{cases} \\
& C_{l} g(x)=\int_{\mathcal{S}^{n-1}} e(x-y) n(y) g(y) d \mathcal{S}_{y}^{n-1}= \begin{cases}g(x), & x \in \mathcal{B}^{n} \\
0, & x \notin \overline{\mathcal{B}^{n}}\end{cases}
\end{aligned}
$$

These results can be generalized to any strongly Lipschitz and bounded open subset $\Omega$ of $\mathbb{R}^{n}$ with boundary $\partial \Omega$ and exterior unit normal $n(y)$ defined for almost all $y \in \partial \Omega$. For details see [9], [8] or [7].

As an immediate consequence we find that both left and right monogenics can be represented in terms of Cauchy integrals.

3. Conformal mappings of the unit ball. It is well known that a complex-valued conformal mapping preserving the unit disk $\mathcal{B}^{2}$ can be writ- 
ten as the composition of a Möbius transformation of type

$$
\varphi_{a}(z)=\frac{z-a}{1-\bar{a} z}, \quad|a|<1,
$$

with a rotation $w=e^{i \phi} z$, with $0 \leq \phi<2 \pi$. The Möbius transformation $\varphi_{a}$ can be characterized as the unique conformal transformation which preserves the unit disk, maps $a$ to the origin and its derivative satisfies $\varphi_{a}^{\prime}(a)>0$. It can be proved that $\varphi_{a} \circ \varphi_{-a}$ is the identity so that $\mathcal{G}(\mathbb{C})=\left\{\varphi_{a}:|a|<1\right\}$ (endowed with the composition) is identified with a subgroup of $\mathrm{SL}(2, \mathbb{C})$, the special linear group. This particular subgroup has the property of leaving the 2-dimensional Laplacian invariant.

Moreover, the derivative of (2) satisfies

$$
\varphi_{a}^{\prime}(z)=\frac{1-a \bar{a}}{(1-\bar{a} z)^{2}}=\frac{1-|a|^{2}}{(1-\bar{a} z)^{2}},
$$

hence proving the invariance of the Poincare metric in the complex disk

$$
\frac{1}{2}|d s|=\frac{|d z|}{1-|z|^{2}}=\frac{\left|d \varphi_{a}(z)\right|}{1-\left|\varphi_{a}(z)\right|^{2}}
$$

under the action of this subgroup.

The higher dimensional counterpart of (2) is the transform

$$
\varphi_{a}(x)=(x-a)(1-\bar{a} x)^{-1}=\frac{(x-a)(1-\bar{x} a)}{|1-\bar{a} x|^{2}},
$$

where $x, a$ are vectors of $\mathcal{B}^{n}$, the $n$-dimensional unit ball (see e.g. [1], [3]). In what follows we shall use the Möbius transformations defined via the right inverse of $1-\bar{a} x$. By duality, analogous results hold for Möbius transformations defined via the left inverse. As in the complex case we have (see [3], [4])

COROLlaRY 3.1. Every conformal transformation $\chi$ leaving the unit ball invariant can be written as the composition of a Möbius transformation of type $\varphi_{a}$ with an orthogonal transformation. Moreover, if $\chi$ is direct then the orthogonal transformation is a rotation.

Several properties are directly extendable to the $n$-dimensional case. In fact, simple calculations show that

$$
1-\left|\varphi_{a}(x)\right|^{2}=\frac{\left(1-|a|^{2}\right)\left(1-|x|^{2}\right)}{|1-\bar{a} x|^{2}}
$$

proving that the group $\mathcal{G}\left(\mathbb{R}^{n}\right)$ of transformations of type (3) preserves the unit ball. This, combined with the relation between the differentials

$$
\left|d \varphi_{a}(x)\right|=\frac{1-|a|^{2}}{|1-\bar{a} x|^{2}}|d x|,
$$


where $|d x|=\sqrt{d x_{1}^{2}+\cdots+d x_{n}^{2}}$, proves the invariance of the $n$-dimensional Poincaré metric

$$
|d s|=\frac{2|d x|}{1-|x|^{2}}=\frac{2\left|d \varphi_{a}(x)\right|}{1-\left|\varphi_{a}(x)\right|^{2}}
$$

under the action of $\mathcal{G}\left(\mathbb{R}^{n}\right)$. Therefore, the change of variable $y=\varphi_{a}(x)$, $|a|<1$, yields for the integral over the unit ball the formula

$$
\int_{\mathcal{B}^{n}} f(y) d y=\int_{\mathcal{B}^{n}} f\left(\varphi_{a}(x)\right)\left(\frac{1-|a|^{2}}{|1-\bar{a} x|^{2}}\right)^{n} d x .
$$

Note that both (3) and the differential form (5) have a singularity at $z_{0}=a /|a|^{2}$. However, there is an important difference between the complex and the $n$-dimensional versions of the group preserving the unit ball. While in $\mathbb{C}$ this group preserves the Laplacian, in the sense that

$$
\Delta\left(f \circ \varphi_{a}\right)=(\Delta f) \circ \varphi_{a}, \quad \forall \varphi_{a} \in \mathcal{G}(\mathbb{C}),
$$

this is no longer true in higher dimensions. On the other hand, the group $\mathcal{G}\left(\mathbb{R}^{n}\right)$ preserves the Laplace-Beltrami operator

$$
\Delta_{\mathrm{LB}} f=\operatorname{div} \operatorname{grad} f=\frac{1}{\sqrt{|g|}} \sum_{i=1}^{n} \frac{\partial}{\partial x_{i}}\left(\sqrt{|g|} \sum_{j=1}^{n} g^{i j} \frac{\partial f}{\partial x_{j}}\right),
$$

related to the metric tensor $d s^{2}=\sum_{i, j} g_{i j} d x_{i} d x_{j}$, where $\left(g^{i j}\right)$ stands for the inverse matrix of $\left(g_{i j}\right)$ and $|g|=\operatorname{det}\left(g_{i j}\right)$. In the present case, we have for the Laplace-Beltrami operator the expression

$$
\Delta_{\mathrm{LB}} f=\left(1-r^{2}\right)\left[\left(1-r^{2}\right) \Delta+2(n-2) r \partial_{r}\right] f,
$$

and it satisfies the invariance condition

$$
\Delta_{\mathrm{LB}}\left(f \circ \varphi_{a}\right)=\left(\Delta_{\mathrm{LB}} f\right) \circ \varphi_{a}
$$

for all $\varphi_{a}$ in $\mathcal{G}\left(\mathbb{R}^{n}\right)$. The solutions of the Laplace-Beltrami operator are called hyperbolically harmonic functions or $\mathcal{M}$-harmonic functions.

4. Poisson and Poisson-Szegô kernels for $\mathcal{B}^{n}$. In the two-dimensional case, it is well known that the Poisson and Poisson-Szegó kernels coincide, both integrals being conformally invariant. As a consequence, harmonic complex-valued functions are uniquely reconstructed from their values on the unit sphere.

In higher dimensions, however, that is no longer true, since the Laplacian is no longer a conformally invariant operator; therefore, the Poisson kernel, which is defined by means of the outward derivative of the Green function for the Laplacian in the unit ball $\mathcal{B}^{n}$, does not coincide with the Poisson-Szegó kernel, a positive conformally invariant kernel arising out of the Szegó kernel 
for the unit ball. Moreover, both the Poisson-Szegó and the Szegó kernels are connected to the Laplace-Beltrami operator (6).

In fact, let $G(x, y)$ be the Green's function for the Laplacian with respect to the unit ball $\mathcal{B}^{n}$. This function is uniquely determined by the following properties:

- it is smooth for all $x \in \mathcal{B}^{n}, y \in \overline{\mathcal{B}^{n}}$ such that $x \neq y$;

- $\Delta_{y} G(x, y)=\delta(x-y)$;

- for a fixed $x \in \mathcal{B}^{n}, G(x, y)-\frac{1}{\omega_{n}|x-y|^{n-2}}$ is harmonic in the $y$ variable;

- $G(x, y)=0$ for all $x \in \mathcal{B}^{n}$ and $y \in \mathcal{S}^{n-1}$.

Denoting by $n_{y}=y$ the outward unit normal to $\mathcal{S}^{n-1}$ at $y$ we have the Poisson kernel in the unit ball

$$
P(x, y)=-\frac{\partial G(x, y)}{\partial n_{y}}=\frac{1}{\omega_{n}} \frac{1-|x|^{2}}{|1-\bar{x} y|^{n}},
$$

defined in $\mathcal{B}^{n} \times \mathcal{S}^{n-1}$. Note that $P$ has a singularity of the same order as the one of the fundamental solution of the Laplace operator.

Now, let us define the Szegö kernel in $\mathcal{B}^{n}$. From the differential relation (5) we derive the change of coordinates for the surface element $d \mathcal{S}^{n-1}$ on $\mathcal{S}^{n-1}$ (unique rotationally invariant measure on the unit sphere) as

$$
\int_{\mathcal{S}^{n-1}} d \mathcal{S}_{\varphi_{a}(x)}^{n-1}=\int_{\mathcal{S}^{n-1}}\left(\frac{1-|a|^{2}}{|1-\bar{a} x|^{2}}\right)^{n-1} d \mathcal{S}_{x}^{n-1}
$$

leading to the following expression for the (positive) Poisson-Szegó kernel in $\mathcal{B}^{n}$ (which is related to the Szegó kernel $S(x, \xi)$ ):

$$
P S(x, \xi)=\left(\frac{1-|x|^{2}}{|1-\bar{x} \xi|^{2}}\right)^{n-1}=\frac{|S(x, \xi)|^{2}}{S(x, x)},
$$

hence, for this last kernel we have

$$
S(x, \xi)=\left(\frac{1-\bar{\xi} x}{|1-\bar{x} \xi|^{2}}\right)^{n-1} .
$$

The behaviour of the Szegó kernel under the action of $\mathcal{G}\left(\mathbb{R}^{n}\right)$ is given by

$$
S\left(\varphi_{a}(x), \varphi_{a}(\xi)\right)=\left(\frac{1}{1-|a|^{2}} \frac{(1-\bar{a} \xi)(1-\bar{\xi} x)(1-\bar{x} a)}{|1-\bar{x} \xi|^{2}}\right)^{n-1} .
$$

Moreover,

$$
\left|S\left(\varphi_{a}(x), \varphi_{a}(\xi)\right)\right|=\frac{|S(a, a)|}{|S(a, \xi)||S(x, a)|}|S(x, \xi)|,
$$

and in the particular case of $\xi=x$ we have $(1-\bar{a} x)(1-\bar{x} x)(1-\bar{x} a) \in \mathbb{R}$. Therefore,

$$
S\left(\varphi_{a}(x), \varphi_{a}(x)\right)=\frac{S(a, a) S(x, x)}{|S(a, x)|^{2}} .
$$


These results lead to the transformation rule for the Poisson-Szegó kernel

$$
\begin{aligned}
P S\left(\varphi_{a}(x), \varphi_{a}(\xi)\right) & =\frac{\left|S\left(\varphi_{a}(x), \varphi_{a}(\xi)\right)\right|^{2}}{S\left(\varphi_{a}(x), \varphi_{a}(x)\right)}=\frac{S(a, a)}{|S(a, \xi)|^{2}} \frac{|S(x, \xi)|^{2}}{S(x, x)} \\
& =\frac{P S(x, \xi)}{P S(a, \xi)} .
\end{aligned}
$$

Now, we obtain the following important result:

THEOREM 4.1. For each fixed $x \in \mathcal{B}^{n}$ the measure $d \mu_{x}(\xi)$ on the boundary $\mathcal{S}^{n-1}$ given by

$$
d \mu_{x}(\xi)=P S(x, \xi) d \mathcal{S}^{n-1}(\xi)
$$

is invariant under the transformations of type (3), that is,

$$
d \mu_{\varphi_{a}(x)}\left(\varphi_{a}(\xi)\right)=d \mu_{x}(\xi)
$$

Proof. Using relation (5) we have

$$
d \mathcal{S}^{n-1}\left(\varphi_{a}(\xi)\right)=\left(\frac{1-|a|^{2}}{|1-\bar{a} \xi|^{2}}\right)^{n-1} d \mathcal{S}^{n-1}(\xi) .
$$

Now it is easily seen that

$$
\begin{aligned}
P S\left(\varphi_{a}(x), \varphi_{a}(\xi)\right) d \mathcal{S}^{n-1}\left(\phi_{a}(\xi)\right) & =\frac{P S(x, \xi)}{P S(a, \xi)}\left(\frac{1-|a|^{2}}{|1-\bar{a} \xi|^{2}}\right)^{n-1} d \mathcal{S}^{n-1}(\xi) \\
& =P S(x, \xi) d \mathcal{S}^{n-1}(\xi)
\end{aligned}
$$

A simple calculation shows that $-D D=\Delta$, where $\Delta$ denotes the Laplacian in $\mathbb{R}^{n}$. Therefore, one can regard monogenic functions as an analogue to holomorphic functions. The linkage of monogenic functions with harmonic functions tells us that every monogenic function is harmonic. It is well-known that harmonic functions can be represented by Poisson integrals. Unfortunately, in higher dimensions the Poisson integral is no longer invariant under conformal transformations. This leads to the consideration of hyperbolically harmonic functions which are not only conformally invariant but can be represented by Poisson-Szegó integrals.

5. Carleson measures. In this section we shall consider the space of all real-valued $f \in L_{p}\left(\mathcal{S}^{n-1}\right), p \geq 1$, such that the Poisson-Szegó transform

$$
\mathcal{P S}[f](x)=\int_{\mathcal{S}^{n-1}} f(\xi) P S(x, \xi) d \mathcal{S}^{n-1}(\xi), \quad|x|<1,
$$

is a hyperbolically harmonic function. By abuse of language we shall denote henceforth these spaces as $h^{p}$, that is,

$$
\begin{aligned}
h^{p}=\left\{f \in L_{p}\left(\mathcal{S}^{n-1}\right):\left(\exists g: \mathcal{B}^{n} \rightarrow \mathcal{C l}_{0, n}\right)\right. & \Delta_{\mathrm{LB}} g=0 \\
& \left.\& \sup _{0 \leq r<1}\|g(r \cdot)\|_{L_{p}\left(\mathcal{S}^{n-1}\right)}<\infty\right\} .
\end{aligned}
$$


For the consistence of such a construction, we refer to [10, Theorem 3.3.4(b) and Theorem 4.4.3]. Finally, we shall prove that for every $f \in h^{p}$, its $L_{p}$-norm in the unit ball (with respect to a given measure $\nu$ ) can be estimated in terms of its $L_{p}$-norm on the unit sphere if $\nu$ is a Carleson measure.

Definition 5.1. A positive measure $\nu$ defined on the unit ball $\mathcal{B}^{n}$ in $\mathbb{R}^{n}$ is a $p$-Carleson measure if there exists a constant $A>0$ such that

$$
\nu\left(Q_{h}(\xi)\right) \leq A h^{(n-1) p}
$$

for any subset

$$
Q_{h}(\xi):=\left\{x \in \mathcal{B}^{n}:|x-\xi|<h\right\},
$$

where $h>0$ and $\xi \in \mathcal{S}^{n-1}$.

In the case of complex functions defined on the unit circle we make use of a Carleson box

$$
\left\{z=r e^{i \theta}: 1-h \leq r<1, \theta_{0} \leq \theta \leq \theta_{0}+h\right\}
$$

instead of $Q_{h}(\xi)$.

The importance of Carleson measures is highlighted in the theorems to be proved later in this section. We will also need a covering lemma which can be found in [11].

Lemma 5.2. Let $F=\left\{B\left(x_{\sigma}, r_{\sigma}\right): \sigma \in \Lambda\right\}$ be a family of balls with bounded radii. Then for each constant $c>0$ there exists a countable subfamily $\left\{B\left(x_{i}, r_{i}\right): i \in \mathbb{N}\right\}$ consisting of pairwise disjoint balls such that each ball in $F$ is contained in one of the balls $B\left(x_{i}, c r_{i}\right)$.

We define the surface ball $E(x, r), x \in \mathcal{S}^{n-1}$ and $r>0$, as the intersection of the ball $B(x, r)$ with the unit sphere $\mathcal{S}^{n-1}$. For each $a \in \mathcal{B}^{n}$ we now consider the particular family of surface balls

$$
\begin{aligned}
E^{m}(a) & :=E\left(a /|a|, 2^{m}(1-|a|)\right) \\
& =B\left(a /|a|, 2^{m}(1-|a|)\right) \cap \mathcal{S}^{n-1}, \quad m=0,1,2, \ldots,
\end{aligned}
$$

with $E^{m}(a)=\mathcal{S}^{n-1}$ for all $m \geq M$, where $M$ is the smallest natural number satisfying $2^{M}(1-|a|) \geq 2$ (see Figure 1 ). Moreover, it can be proved that this family has the following additional properties (see [2]):

$$
\frac{C(n) 2^{n-2}(1-|a|)^{n-1}}{(n-1) \pi^{n-2}} \leq \mu\left(E^{0}(a)\right) \leq \frac{C(n) 2^{n-1}(1-|a|)^{n-1}}{n-1}
$$

and

$$
\mu\left(E\left(x_{0}, t r\right)\right) \leq c t^{n-1} \mu\left(E\left(x_{0}, r\right)\right)
$$

from which it follows that $\mu\left(E^{m}(a)\right) \leq c 2^{m(n-1)} \mu\left(E^{0}(a)\right)$. 


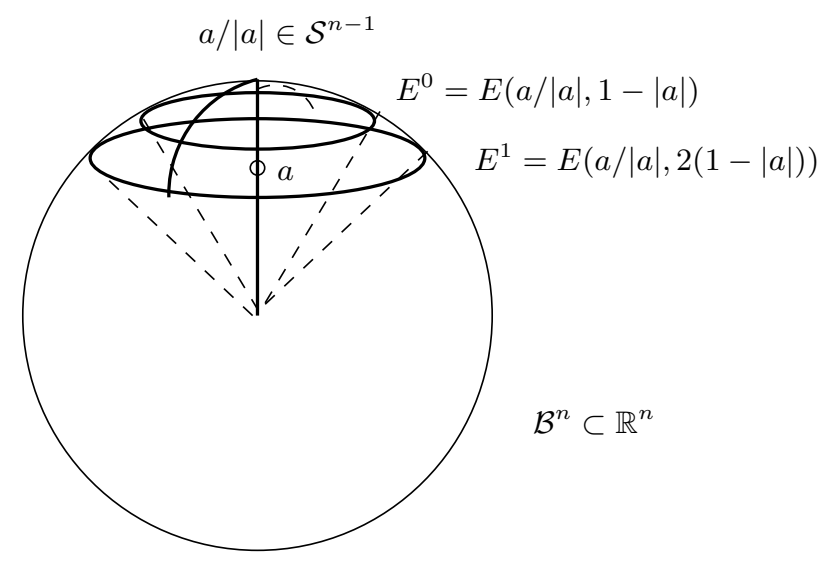

Fig. 1. Surface balls

The following theorem provides a necessary and suficient condition for a positive measure to be a Carleson measure, given in terms of the PoissonSzegó kernel.

Theorem 5.3. A positive measure $\nu$ defined on the unit ball $\mathcal{B}^{n}$ is a $p$-Carleson measure if and only if

$$
\sup _{a \in \mathcal{B}^{n}} \int_{\mathcal{B}^{n}}\left(\frac{1-|a|^{2}}{|1-\bar{a} x|^{2}}\right)^{(n-1) p} d \nu<\infty
$$

Proof. In a first step we assume

$$
\sup _{a \in \mathcal{B}^{n}} \int_{\mathcal{B}^{n}}\left(\frac{1-|a|^{2}}{|1-\bar{a} x|^{2}}\right)^{(n-1) p} d \nu<C_{p}<\infty
$$

For all $a \in \mathcal{B}^{n}$ we write $a=|a| \frac{a}{|a|}=(1-\delta) \xi$, where $\xi \in \mathcal{S}^{n-1}$ and $0<\delta<1$. Then for all $x \in Q_{\delta}(\xi)$ we have

$$
|1-\bar{a} x|=|1-\bar{\xi} x+\delta \bar{\xi} x| \leq|1-\bar{\xi} x|+\delta|x|=|\xi-x|+\delta|x|<\delta+\delta|x| \leq 2 \delta
$$
and, on the other hand,

$$
1-|a|^{2}=1-(1-\delta)^{2}=2 \delta-\delta^{2}=\delta(2-\delta) \geq \delta .
$$

Therefore, we obtain the estimate

$$
\nu\left(Q_{\delta}(\xi)\right) \leq\left(\frac{(2 \delta)^{2}}{\delta}\right)^{(n-1) p} \int_{\mathcal{B}^{n}}\left(\frac{1-|a|^{2}}{|1-\bar{a} x|^{2}}\right)^{(n-1) p} d \nu \leq 4^{(n-1) p} C_{p} \delta^{(n-1) p} .
$$

This ends the first part of the proof.

Conversely, we now assume $\nu$ to be a Carleson measure, i.e.

$$
\sup _{\delta, \xi} \frac{\nu\left(Q_{\delta}(\xi)\right)}{\delta^{(n-1) p}}=K(n, p)<\infty,
$$

and therefore $\nu$ is a finite measure. 
We first assume $|a| \leq 3 / 4$, so that

$$
|1-\bar{a} x| \geq 1-\frac{3}{4}|x| \geq \frac{1}{4} .
$$

Note that $\mathcal{B}^{n} \subseteq Q_{\delta}(\xi)$ for all $\delta \geq 2$. Now

$$
\int_{\mathcal{B}^{n}} d \nu \leq \nu\left(Q_{2}(\xi)\right) \leq 2^{(n-1) p} K(n, p),
$$

and we obtain

$$
\begin{aligned}
\sup _{|a| \leq 3 / 4} \int_{\mathcal{B}^{n}}\left(\frac{1-|a|^{2}}{|1-\bar{a} x|^{2}}\right)^{(n-1) p} d \nu & \leq 4^{2(n-1) p} \sup _{|a| \leq 3 / 4} \int_{\mathcal{B}^{n}} d \nu \\
& \leq 2^{4(n-1) p} \nu\left(Q_{2}(\xi)\right) \\
& \leq 2^{5(n-1) p} K(n, p)<\infty .
\end{aligned}
$$

It remains to consider the case of $|a|>3 / 4$. We define for $\xi=a /|a|$ the family of sets

$$
F_{0}=Q_{2(1-|a|)}(\xi) \text { and } \quad F_{k}=Q_{2^{k+1}(1-|a|)}(\xi) \backslash Q_{2^{k}(1-|a|)}(\xi) .
$$

Hence, we get

$$
\nu\left(F_{k}\right) \leq \nu\left(Q_{2^{k+1}(1-|a|)}(\xi)\right) \leq K(n, p)\left(2^{k+1}(1-|a|)\right)^{(n-1) p} .
$$

Furthermore,

$$
\begin{aligned}
|1-\bar{a} x| & =\left|\frac{a}{|a|}-\right| a|x|=|\xi-x+x-| a|x| \\
& \geq|\xi-x|-(1-|a|)|x| \geq 2^{k}(1-|a|)-(1-|a|)|x| \\
& \geq 2^{k-1}(1-|a|), \quad \forall x \in F_{k}, \quad k \geq 1,
\end{aligned}
$$

while for $x \in F_{0}$ we have $1 / 2<|x| \leq 1$ so that

$$
|1-\bar{a} x| \geq 1-|a||x|=(1-|x|)+|x|(1-|a|) \geq \frac{1}{2}(1-|a|),
$$

thus proving the validity of estimate (15) for all $k \geq 0$. We then obtain

$$
\begin{aligned}
\int_{F_{k}}\left(\frac{1-|a|^{2}}{|1-\bar{a} x|^{2}}\right)^{(n-1) p} d \nu & \leq \frac{(1+|a|)^{(n-1) p}(1-|a|)^{(n-1) p}}{\left(2^{k-1}(1-|a|)\right)^{2(n-1) p}} \int_{F_{k}} d \nu \\
& \leq \frac{2^{p(n-1)(3-2 k)}}{(1-|a|)^{(n-1) p}} K(n, p) 2^{p(n-1)}(1-|a|)^{(n-1) p} \\
& =K(n, p) 2^{2 p(n-1)(2-k)}
\end{aligned}
$$


and we conclude the proof with

$$
\begin{aligned}
\sup _{|a|>3 / 4} \int_{\mathcal{B}^{n}}\left(\frac{1-|a|^{2}}{|1-\bar{a} x|^{2}}\right)^{(n-1) p} d \nu & =\sup _{|a|>3 / 4} \sum_{k=0}^{\infty} \int_{F_{k}}\left(\frac{1-|a|^{2}}{|1-\bar{a} x|^{2}}\right)^{(n-1) p} d \nu \\
& \leq K(n, p) 2^{4 p(n-1)} \sum_{k=0}^{\infty}\left(2^{-2 p(n-1)}\right)^{k}<\infty
\end{aligned}
$$

Finally, we present a necessary criterion for a positive measure to be a Carleson measure, based on estimates of the $L_{p}$-norm of the boundary values of $f \in h^{p}$. For that purpose, we first prove the following result regarding an estimation of the values of a hyperbolically harmonic function by means of its boundary values.

THEOREM 5.4. For every $f \in h^{1}$,

$$
|f(a)| \lesssim \widetilde{f}(a)+\|f\|_{1}, \quad \forall|a|<1
$$

where

$$
\tilde{f}(a):=\sup _{m=0,1, \ldots, M} \frac{1}{\mu\left(E^{m}(a)\right)} \int_{E^{m}(a)}|f(y)| d \mathcal{S}_{y}^{n-1} .
$$

Proof. Under the theorem's conditions we have (see e.g. [5, p. 34] for a proof in the harmonic complex case)

$$
|f(a)| \leq \int_{\mathcal{S}^{n-1}} P S(a, y)|f(y)| d \mathcal{S}_{y}^{n-1}
$$

For simplicity, write $\theta=\angle(x, a)$. For each $x \in \mathcal{S}^{n-1}$ and $a \in \mathcal{B}^{n}$ we have the initial estimate

$$
\frac{1-|a|^{2}}{1+|a|^{2}-2|a| \cos \theta} \leq \frac{1+|a|}{1-|a|} \leq \frac{2}{1-|a|},
$$

which can be improved, for $|a| \geq 1 / 2$ and $x \in E^{m+1}(a) \backslash E^{m}(a)$, to

$$
\frac{1-|a|^{2}}{1+|a|^{2}-2|a| \cos \theta} \leq \frac{2^{2-2 m}}{1-|a|}
$$

Applying the Poisson-Szegó formula and inequality (9) we obtain, for $|a| \geq 1 / 2$,

$$
\begin{aligned}
& |f(a)| \leq \frac{1}{\omega_{n}} \int_{\mathcal{S}^{n-1}}|f|\left(\frac{1-|a|^{2}}{1+|a|^{2}-2|a| \cos \theta}\right)^{n-1} d \mathcal{S}^{n-1} \\
& \leq \frac{1}{\omega_{n}}\left[\int_{E^{0}(a)} \frac{2^{n-1}|f|}{(1-|a|)^{n-1}} d \mathcal{S}^{n-1}+\sum_{m=0}^{M-1} \int_{E^{m+1}(a) \backslash E^{m}(a)} \frac{2^{2(n-1)(1-m)}|f|}{(1-|a|)^{n-1}} d \mathcal{S}^{n-1}\right]
\end{aligned}
$$




$$
\begin{aligned}
\leq & \frac{1}{\omega_{n}}\left[\frac{C(n) 2^{2(n-1)}}{\mu\left(E^{0}(a)\right)(n-1)} \int_{E^{0}(a)}|f| d \mathcal{S}^{n-1}\right. \\
& \left.+\sum_{m=0}^{M-1} \frac{c 2^{(m+1)(n-1)} C(n) 2^{n-1}}{\mu\left(E^{m+1}(a)\right)(n-1)} \int_{E^{m+1}(a) \backslash E^{m}(a)} 2^{2(n-1)(1-m)}|f| d \mathcal{S}^{n-1}\right] \\
= & \frac{C(n) 2^{2(n-1)}}{(n-1) \omega_{n}}\left[\frac{1}{\mu\left(E^{0}(a)\right)} \int_{E^{0}(a)}|f| d \mathcal{S}^{n-1}\right. \\
& \left.+\sum_{m=0}^{M-1} c 2^{2-m / 2} \frac{1}{\mu\left(E^{m+1}(a)\right)} \int_{E^{m+1}(a)}|f| d \mathcal{S}^{n-1}\right] \\
\leq & \frac{C(n) 2^{2(n-1)}}{(n-1) \omega_{n}}\left(1+4 c \sum_{m=0}^{M-1} 2^{-m / 2}\right) \tilde{f}(a) .
\end{aligned}
$$

In the case of $|a|<1 / 2$, we have the following estimate for the PoissonSzegó kernel:

$$
P S(a, y) \leq\left[\frac{1-|a|^{2}}{(1-|a|)^{2}}\right]^{n-1} \leq 2^{n-1}, \quad y \in \mathcal{S}^{n-1}
$$

and, therefore,

$$
|f(a)| \leq \int_{\mathcal{S}^{n-1}} P S(a, y)|f(y)| d \mathcal{S}^{n-1} \leq \frac{2^{n}}{\omega_{n}} \int_{\mathcal{S}^{n-1}}|f(y)| d \mathcal{S}^{n-1} \leq K\|f\|_{1} .
$$

Combining the above estimates we get the desired result

$$
|f(a)| \lesssim \widetilde{f}(a)+\|f\|_{1} \quad \text { for all }|a|<1 .
$$

THEOREM 5.5. If the positive measure $\nu$ defined on the unit ball $\mathcal{B}^{n}$ is a Carleson measure then for all $1<p<\infty$ there exists a constant $C>0$ such that

$$
\left(\int_{\mathcal{B}^{n}}|f(x)|^{p} d \nu(x)\right)^{1 / p} \leq C\|f\|_{p} \quad \text { for all } f \in h^{p} .
$$

Proof. In a first step, we shall prove that the mapping $f \mapsto \widetilde{f}$ is of weak type $(1,1)$, i.e. the Carleson measure of the set $M_{s}:=\left\{x \in \mathcal{B}^{n}: \widetilde{f}(x)>s\right\}$ satisfies

$$
\nu\left(M_{s}\right) \lesssim s^{-1}\|f\|_{1}
$$

Let $K_{s}$ be a compact subset of $M_{s}$. Consider the covering of $K_{s}$ by the family of balls $B(x /|x|, r)$, where $x \in K_{s}$ and $r=2(1-|x|)$. Note that

- $B(x /|x|, r) \cap \mathcal{S}^{n-1}=E(x /|x|, r)$;

- $\left|\frac{x}{|x|}-x\right|=\left|\frac{x}{|x|}(1-|x|)\right|=(1-|x|)$, hence $x \in B(x /|x|, r)$. 
Due to Lemma 5.2 (we will assume the constant $c=4$ ) and because $K_{s}$ is compact we can extract a finite subfamily $\left\{B\left(x_{i} /\left|x_{i}\right|, r_{i}\right): i=1, \ldots, l\right\}$ satisfying

$$
\bigcup_{i=1}^{l} B\left(x_{i} /\left|x_{i}\right|, r_{i}\right) \cap \mathcal{B}^{n} \subseteq K_{s} \subseteq \bigcup_{i=1}^{l} B\left(x_{i} /\left|x_{i}\right|, 4 r_{i}\right) \cap \mathcal{B}^{n} .
$$

In accordance with Definition 5.1 we write $Q_{r_{i}}\left(x_{i} /\left|x_{i}\right|\right)=B\left(x_{i} /\left|x_{i}\right|, r_{i}\right) \cap \mathcal{B}^{n}$. Since $\nu$ is a Carleson measure we have

$$
\begin{aligned}
\nu\left(K_{s}\right) & \leq \nu\left(\bigcup_{i=1}^{l} Q_{4 r_{i}}\left(x_{i} /\left|x_{i}\right|\right)\right) \leq \sum_{i=1}^{l} \nu\left(Q_{4 r_{i}}\left(x_{i} /\left|x_{i}\right|\right)\right) \\
& \leq A \sum_{i=1}^{l}\left(4 r_{i}\right)^{n-1}=A 8^{n-1} \sum_{i=1}^{l}\left(1-\left|x_{i}\right|\right)^{n-1}
\end{aligned}
$$

Due to estimate $(9)$, we get

$$
A 8^{n-1} \sum_{i=1}^{l}\left(1-\left|x_{i}\right|\right)^{n-1} \leq \frac{A(n-1) \pi^{n-2}}{2^{1-2 n} C(n)} \sum_{i=1}^{l} \mu\left(E^{0}\left(x_{i}\right)\right) .
$$

For each $x_{i} \in K_{s} \subset M_{s}$ we have

$$
\begin{gathered}
s<\sup _{m=0, \ldots, M} \frac{1}{\mu\left(E^{m}\left(x_{i}\right)\right)} \int_{E^{m}\left(x_{i}\right)}|f| d \mathcal{S}^{n-1}=\widetilde{f}\left(x_{i}\right), \\
\mu\left(E^{0}\left(x_{i}\right)\right) \leq \frac{1}{s} \sup _{m=0, \ldots, M} \frac{\mu\left(E^{0}\left(x_{i}\right)\right)}{\mu\left(E^{m}\left(x_{i}\right)\right)} \int_{E^{m}\left(x_{i}\right)}|f| d \mathcal{S}^{n-1} \leq \frac{1}{s}\|f\|_{1} .
\end{gathered}
$$

Inserting now this estimate in (19), we obtain

$$
\begin{aligned}
\nu\left(K_{s}\right) & \leq \frac{A(n-1) \pi^{n-2}}{2^{1-2 n} C(n)} \sum_{i=1}^{l} \mu\left(E\left(x_{i} /\left|x_{i}\right|, 1-\left|x_{i}\right|\right)\right) \\
& <\frac{A(n-1) \pi^{n-2}}{2^{1-2 n} C(n)} \frac{l}{s}\|f\|_{1}
\end{aligned}
$$

and, therefore, $\nu\left(M_{s}\right)=\sup _{K_{s} \subset M_{s}} \nu\left(K_{s}\right) \lesssim s^{-1}\|f\|_{1}$, which concludes the first part of the proof.

In a second step, we shall prove that the mapping $f \mapsto \widetilde{f}$ is of weak type $(p, p)$ for $p>1$, that is, $\nu\left(M_{s}\right) \lesssim\|f\|_{p}^{p}$. For that we introduce the auxiliary function

$$
f_{1}(x)= \begin{cases}f(x), & |f(x)|>s / 2 \\ 0, & \text { otherwise }\end{cases}
$$


and we obtain

$$
\frac{s}{2}<\sup _{m=0, \ldots, M} \frac{1}{\mu\left(E^{m}(x)\right)} \int_{E^{m}(x)}\left|f_{1}(y)\right| d \mathcal{S}_{y}^{n-1} \leq \widetilde{f}(x),
$$

that is, $M_{s} \subset \widetilde{M}_{s / 2}=\left\{x \in \mathcal{B}^{n}: \widetilde{f}_{1}>s / 2\right\}$. Therefore, $\nu\left(M_{s}\right) \leq \nu\left(\widetilde{M}_{s / 2}\right)$ implies

$$
\nu\left(M_{s}\right) \leq c\left\|f_{1}\right\|_{1} \leq \frac{c}{s} \int_{\mathcal{S}^{n-1}}\left|f_{1}\right| d \mathcal{S}^{n-1}=\frac{c}{s} \int_{\left\{x \in \mathcal{S}^{n-1}:|f|>s / 2\right\}}|f| d \mathcal{S}^{n-1} .
$$

Using (18) we have

$$
s^{p-1} \nu\left(M_{s}\right) \leq C s^{p-2}\|f\|_{1},
$$

which leads to

$$
\begin{aligned}
\int_{0}^{2|f|} s^{p-1} \nu\left(M_{s}\right) d s & \leq C \int_{0}^{2|f|} s^{p-2}\|f\|_{1} d s=C \int_{\mathcal{S}^{n-1}}|f| \int_{0}^{2|f|} s^{p-2} d s d \mathcal{S}^{n-1} \\
& =C \frac{2^{p-1}}{p-1} \int_{\mathcal{S}^{n-1}}|f|^{p} d \mathcal{S}^{n-1}=C \frac{2^{p-1}}{p-1}\|f\|_{p}^{p}
\end{aligned}
$$

for all $p>1$. Since $\tilde{f}$ is $\nu$-measurable in $M_{s}$ we now apply the formula

$$
\frac{s^{p}}{p} \nu\left(M_{s}\right) \leq \frac{1}{p} \int_{M_{s}}|\tilde{f}|^{p} d \nu=\int_{0}^{2|f|} s^{p-1} \nu\left(M_{s}\right) d s
$$

to obtain the desired result.

By the Marcinkiewicz interpolation theorem ([10]), we conclude that the mapping $f \mapsto \widetilde{f}$ is of strong type $(p, p)$ for all $1<p<\infty$, that is,

$$
\|\widetilde{f}\|_{\nu, p}^{p}=\int_{\mathcal{B}^{n}}|\widetilde{f}|^{p} d \nu \lesssim\|f\|_{p}^{p}
$$

Finally, we conclude our proof with the help of Theorem 5.4 and of (20). In fact,

$$
\begin{aligned}
\left(\int_{\mathcal{B}^{n}}|f|^{p} d \nu\right)^{1 / p} & =\|f\|_{\nu, p} \leq K\|\tilde{f}+\| f\left\|_{1}\right\|_{\nu, p} \\
& \leq K\left(\|\widetilde{f}\|_{\nu, p}+\|f\|_{1} \nu\left(\mathcal{B}^{n}\right)\right) \lesssim\|f\|_{p}
\end{aligned}
$$

since $\|f\|_{1} \leq\|f\|_{p}$ for $p>1$.

6. Conclusion. We must remark that this paper leaves several open questions. First, it remains to establish a generalization of the classical harmonic Hardy spaces to $\mathrm{Cl}_{0, n}$-valued functions. Second, one should investigate the possible connection between these spaces and the classical harmonic $h^{p}$, as well as its relation to already existing monogenic Hardy-type spaces. 


\section{References}

[1] L. V. Ahlfors, Möbius transformations in several dimensions, Ordway Professorship Lectures in Mathematics, Univ. of Minnesota, Minneapolis, MN, 1981.

[2] S. Bernstein, Integralgleichungen und Funktionenräume für Randwerte monogener Funktionen, Habilitation thesis, Freiberg, 2001.

[3] J. Cnops, Spherical geometry and Möbius transformations, in: Clifford Algebras and their Applications in Mathematical Physics, F. Brackx et al. (eds.), Kluwer, Dordrecht, 1993, 75-84.

[4] J. Cnops and R. Delanghe, Möbius invariant spaces in the unit ball, Appl. Anal. 73 (1999), 45-64.

[5] P. L. Duren, Theory of $H^{p}$ Spaces, Pure Appl. Math. 38, New York, Academic Press, 1970.

[6] K. Gürlebeck and W. Sprößig, Quaternionic and Clifford Calculus for Engineers and Physicists, Wiley, Chichester, 1997.

[7] C. Li, A. McIntosh and T. Qian, Clifford algebras, Fourier transforms, and singular convolution operators on Lipschitz surfaces, Rev. Mat. Iberoamericana 10 (1993), $665-721$.

[8] C. Li, A. McIntosh and S. Stephen, Convolution singular integrals on Lipschitz surfaces, J. Amer. Math. Soc. 5 (1992), 455-481.

[9] A. McIntosh, Clifford algebras, Fourier theory, singular integrals, and harmonic functions on Lipschitz domains, in: Clifford Algebras in Analysis and Related Topics, J. Ryan (ed.), CRC Press, Boca Raton, FL, 1996, 33-87.

[10] W. Rudin, Function Theory in the Unit Ball of $\mathbb{C}^{n}$, Grundlehren Math. Wiss. 241, Springer, New York, 1980.

[11] E. Stein and G. Weiss, Introduction to Fourier Analysis on Euclidean Spaces, Princeton Univ. Press, 1971.

Institute of Applied Analysis

University of Mining and Technology

D-09596 Freiberg, Germany

E-mail: swanhild.bernstein@tu-freiberg.de
Departamento de Matemática

Universidade de Aveiro

P-3810-159 Aveiro, Portugal

E-mail: pceres@mat.ua.pt

Received July 26, 2005

Revised version March 14, 2007 